\title{
AN INTEGRATED APPROACH TO BATTLEFIELD SITUATION ASSESSMENT
}

\author{
Yang Fan, Chang Guocen, Duan Tao and Hua Wenjian \\ The Telecommunication Engineering Institute, Air Force Engineering University, Xi'an, \\ Shaanxi 710077,China
}

Abstract: Situation assessment (SA) is the basis for many of the planning activities performed by the battlefield commander and staff. And as a very complex military process, it requires the cooperation of lots of information processing technology. Multi-agents system (MAS) is a useful method to model the complex Command and Control (C2) system. In this paper, we present a multiagents model for situation assessment. The three main components of this model, which are computation, reasoning and communication, were designed in detail by integrating series of new and useful technology. The computation component calculates the Battlefield Initiative; the reasoning component makes the situation prediction; and the communication component gives a help to interchange situation information among the Situation Assessment Agents (SA-Agents).This model can integrate qualitative reasoning, quantitative computing and multi-source communicating as a whole, and give the result of situation assessment and the risk value to take it, which is very useful in the C2 system simulation.

Key words: $\quad$ situation assessment; multi-agents system; command and control

\section{INTRODUCTION}

Situation assessment is the basis for many of the planning activities performed by the battlefield commander and staff. Improved SA may lead to faster, better planning. There is no general definition of situation assessment, but we can give a functional description, that SA is an explanation of battlefield view according to the force disposition, operation ability and 
efficiency of both sides in the battle, a procession of analysis to hostile attempts and operation plans ${ }^{1}$.

As a very complex military information fusion process, SA has no fixed, mature theory. Because we cannot get a satisfied SA by using any single ready-made approach, it is necessary to integrate them to accomplish this task. And there are some methods based on the information fusion theory to get some degree of SA, such as Plate-based or AI-based technology. In our work, we concern that how to create a framework for integration of such methods.

More and more applications have shown the agent to be a valuable software concept with the potential to be more widely used in command and control system modeling ${ }^{2}$. The ability of agents to perform simple tasks autonomously has aroused much interest in the potential military applications. Key characteristics of agents which make them attractive are their:

- Autonomy;

- High-level representation of behavior;

- Flexible behavior;

- Real-time performance;

- Suitability for distributed applications; and

- Ability to work cooperatively in teams.

And the agent as described here is an autonomous piece of software, which has explicit goals or desires to achieve, and is pre-programmed with plans or behaviors to achieve these goals under varying circumstances. For example, when the BDI agent set to work, it pursues its given goals, adopting the appropriate plans, or intentions, according to its current beliefs of the state of the world, so as to perform the role it has been given. To use the MAS in modeling C2 System can integrate much useful technology.

In this paper, we present a multi-agents model for an important military application - situation assessment. And the paper is organized as follows: Section 2 is dedicated to the framework of the SA-Agents. In section 3 to 5 we discuss the three main components of SA-Agent in detail. Finally section 6 concludes.

\section{FRAMEWORK OF SA-AGENT}

SA-Agent is an intelligent program that takes the task of SA. The main purpose of SA-Agents is to make sure that which side takes the advantage of the battlefield under the situation currently, and to foresee how this kind of advantage develops. And the challenge in the structure design of SA-Agent comes to combination of quality and quantity. So there should be three kinds 
of capabilities that SA-Agents must be equipped with, calculating, foreseeing and communicating. According to this, the framework consisted of three components: computation Reasoning and communication; while communication component consisted of advice encoder/decoder and system I/O. The structure of SA-Agent is shown in fig 1.

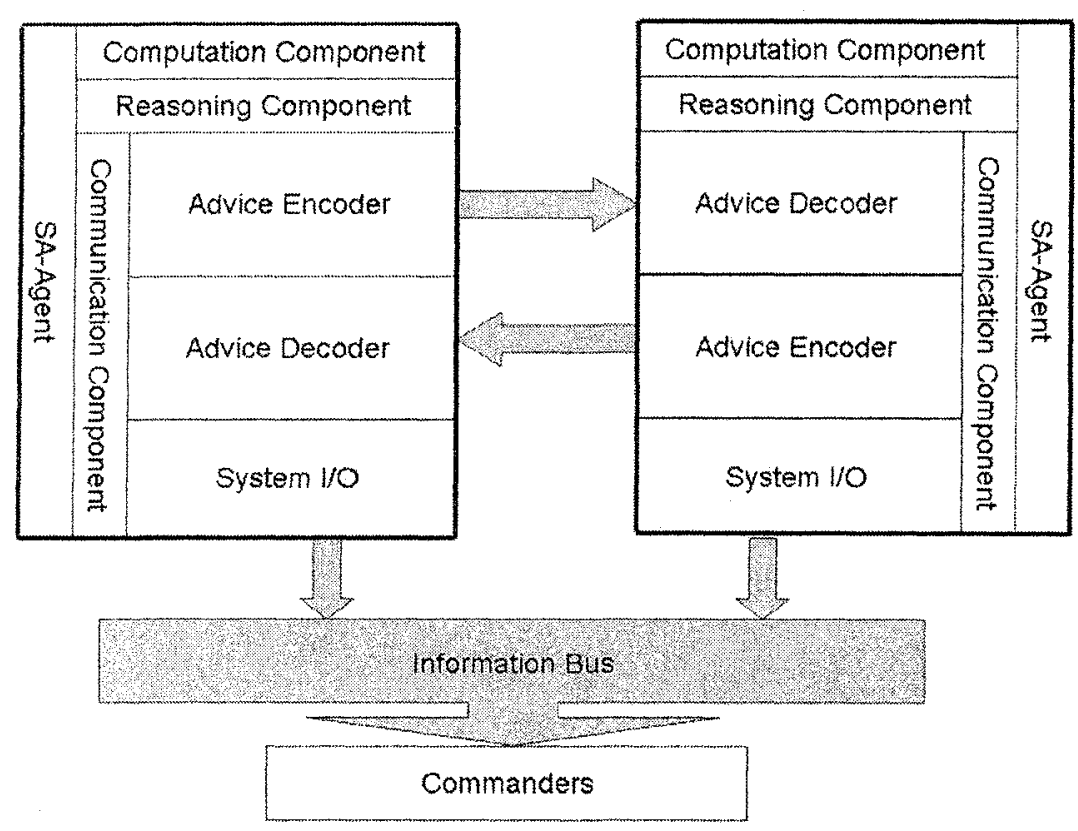

Figure 1. The Framework of SA-Agent

Though there are only two SA-Agents in the Fig.l (limited by the paper), when more SA-Agents join this system, we can get their structures and relationships similarly according to fig 1.In an SA-Agent, Computation Component does the quantitative computing of Battlefield Initiative; Reasoning Component does the qualitative situation reasoning of the battlefield situation development; and Communication Component helps . SA-Agents interchange situation information in the form of Advice. All the three components are discussed in detail in the next three sections. 


\section{COMPUTATION COMPONENT}

One of the most important tasks of SA-Agents is to determine which side takes the advantage of the current battlefield. And SA-Agent uses the BI (Battlefield Initiative) to make the judgment. BI denotes the degree how one side controls the battlefield'.

How the current situation is propitious to realize one side's operation goals is the key to make sure how to calculate the BI. Set the current BI for blue army (one side of the battle, and the contrary side is red army) is $I$, then

$$
I=\sum_{i} k_{i} \times F\left(R_{i}\right) \quad(-1 \leq I \leq 1)
$$

where, If $I=1$, the blue army controls the battlefield completely; or if $I=-1$, the battlefield is completely out of blue army's control.

$F\left(R_{i}\right)$ denotes whether the red can hold back the blue's $i$ th operation plan. SA-Agent calculates $F\left(R_{i}\right)$ following the expression below.

$$
V=X_{i} Y_{i} R_{i} / \sum_{j} L_{j} M_{j} B_{i j}
$$

where $R_{i}$ denotes the potential battle effectiveness of blue's $i$ th operation plan;

$B_{i j}$ denotes the potential battle effectiveness of red's $j$ th operation plan to strike blue's ith operation plan;

$X_{i}\left(L_{,}\right)$reflects the ability of blue (red) 's C2(command and control) System to control the blue's $i$ th (red's $j$ th) operation plan;

$Y_{i}\left(M_{j}\right)$ reflects the ability of blue (red)'s Battlefield Support System to support the blue's $i$ th (red's $j$ th) operation plan;

$\sum_{j} L_{j} M_{j} B_{l j}$ is the actual battle effectiveness of all the red's operation plans to strike the blue's ith operation plan.

$$
F\left(R_{i}\right)=\left\{\begin{array}{cc}
1, & V>U_{U P} \\
0, & U_{\text {DOWN }} \leq V \leq U_{U P} \\
-1, & V<U_{\text {DOHN }}
\end{array}\right.
$$

where $U_{\text {Dow }}, U_{U P}$ are the upper limit and lower limit, which are created by the system and will be adjusted in the practice.

And in (1), $k_{i}$ denotes the contribution of blue's $i$ th operation plan to $I$, the Battlefield Initiative. $k$, reflects the SA-Agent's value judgment to $i$ th operation plan.

$$
K_{,}=R_{i} / \sum_{n} R_{n}
$$

All the parameters related would be obtained by the battlefield sensors system and inputted to the framework through the system I/O before situation assessment begins. The detailed computation methods of (1),(2) are not listed here, because that are beyond this paper's topic. Here, we just set up a kind (maybe not the perfect kind) of computation mechanism with which SA-Agent can get the quantificational Battlefield Initiative. 


\section{REASONING COMPONENT}

Inspired by the Stirling's multiple agent decision-making model ${ }^{3}$, an epistemic system of SA- Agent was designed. Given a situation assessment system, Consider a finite number, $\mathrm{N}$, of SA-Agents, denoted SAAgent ${ }_{i}, \ldots$ SA-Agent ${ }_{\mathrm{N}}$; the epistemic system can be denoted by the triple $\left(\mathrm{K}_{\mathrm{xi}}\right.$, $\mathrm{G}_{\mathrm{xi}}, \mathrm{B}_{\mathrm{xi}}$ ), consisting of the following:

$\mathrm{K}_{\mathrm{xi}}$, a situation knowledge corpus;

$\mathrm{G}_{\mathrm{xi}}$, a system of information valuation;

$B_{x i}$, a system of truth valuation.

A situation prediction is made by considering the informational value of the prediction versus its truth value.

Let $U_{i}$ denote the set of possible situation prediction of SA-Agent $t_{i}$, and assume that $K_{x i}$ entails exactly one element of $U_{i}$, and that all elements of $U_{i}$ are consistent with $\mathrm{K}_{\mathrm{xi}} . \mathrm{U}_{\mathrm{i}}$ is said to be an ultimate patition for $S A$-Agent $\mathrm{i}$. A potential situation prediction for SA-Agent $t_{i}$ occurs if SA-Agent $t_{j}$ is able to reject all and only members of a subset of $U_{i}$ (see more details in 3 ).

But in the SA-Agent reasoning model, unlike the other general agents, the knowledge corpus will keep unchanged until the new situation information is obtained. Because the main purpose of the reasoning component of SA-Agent is to make a prediction for the current situation, the SA-Agent needs not add the "right" prediction to the knowledge corpus. Even SA-Agent will not take any action but prediction, the tradeoff between the utility of avoiding error and the utility of getting more information still exists.

For any $g \subset \mathrm{U}_{\mathrm{i}}$, define the utility of SA-Agent $\mathrm{i}_{\mathrm{i}}$ accepting $\mathrm{g}$ in the interest of avoiding error as $T, g, l)$, with $l \in\{t, f\}$ denoting whether $\mathrm{g}$ is true $(\mathrm{t})$ or false (f), according to $\mathrm{K}_{\mathrm{xi}}$ and $\mathrm{B}_{\mathrm{xi}}$, where

$$
T_{i}(g, l)=\left\{\begin{array}{lll}
1 & \text { if } & l=t \\
0 & \text { if } & l=f
\end{array}\right.
$$

Equation (5) defines the utility of avoiding error, and the utility of getting more information should be defined, too. To assess the utility of information that a situation prediction carries, SA-Agent $\mathrm{i}_{\mathrm{i}}$ may assign an information value to each $g \subset U_{i}$. The potential predictions may be partially ordered with respect to information value. For example, let $\mathrm{g}_{1} \subset \mathrm{U}_{\mathrm{i}}, \mathrm{g} 2 \subset \mathrm{U}_{\mathrm{i}}$ be two potential predictions, if $\widetilde{g}_{1} \subset \widetilde{g}_{2}$, but $\widetilde{g}_{1} \neq \widetilde{g}_{2}$, then $g_{1}$ is less valuable informational than $g_{2}$, because accepting $g_{2}$ rejects more elements of $U_{i}$ than does accepting $g_{1}$.

The informational value of a potential prediction is the sum of the values assigned each element of $U_{i}$ that is rejected according to the potential prediction. Assume $\mathrm{U}_{\mathrm{i}}=\left\{h_{i 1}, h_{i 2}, \ldots ., h_{i i_{i}}\right\}$, and let $M_{i}\left(h_{i j}\right) \geq 0$ denotes the value 
$\mathrm{X}_{\mathrm{i}}$ assigns to $h_{i j}$, according to $\mathrm{G}_{\mathrm{xi}}$, then $\sum_{j=1}^{n_{i=1}} M\left(h i_{j}\right)=1$. For any set $\mathrm{g} \subset \mathrm{U}_{\mathrm{i}}$, define

$$
M_{i}(g)=\sum M_{i}\left(h_{i j}\right)
$$

as the SA-A gentint's informational value of rejecting $g$. And we can define $C_{i}(g)=1-M_{i}(g)$

Then we get the utility function, $T_{i}(g, l)$, of avoiding error and the utility function, $C_{i}(g)$, of getting information. So the epistemic utility function for situation prediction can be defined as a convex combination:

$$
u_{i}(g, l)=\alpha_{i} T_{i}(g, l)+\left(1-\alpha_{i}\right) C_{i}(g)
$$

where the quantity $\alpha_{i}$ represents the relative importance that SA-Agent attaches to avoiding error versus getting more information. Equation (8) is the basis for SA-Agent to make tradeoff in the process of situation prediction. Similar to section 3 , the detailed reasoning process will not be discussed here, but notice that, for the suitability for agent technology in military command and control, $\alpha_{i}$ usually be restrict in $(0.5,1)$.

\section{COMMUNICATION COMPONENT}

In Section 3 and Section 4, we have discussed how a SA-Agent calculates the Battlefield Initiative and reasons out a situation prediction. But the two methods for situation assessment are both for single agent, and we must consider the communication, interaction and coordination among the SA-Agents to make better use of the virtues of MAS. And we do not concern the communication protocol here, instead our focus are the interchange of situation information and the cooperation on the situation prediction.

A SA-Agent must get some computation or reasoning parameters in order to make situation assessment. How to get and send those parameters correctly is the key problem in the Communication Component. As the framework we gave in section 2, SA-Agents send and receive these parameters in the form of Advice ${ }^{4}$. An Advice from SA-Agent $t_{i}$ to SA-Agent ${ }_{j}$ can be designed as follow:

Advice $_{i j}\left(\mathrm{ID}_{-.}\right.$parameter, Truth_parameter, Trust_Agent ${ }_{i}$ )

where ID_ parameter is the unique mark to identify the parameter; Truth parameter is the numerical value that SA-Agent ${ }_{i}$ sends to SA-Agent; ;rust $_{\text {- }}$ parameter is the Trust of SA-Agent ${ }_{i}$. And every SA-Agent has an encoder/decoder in its communication component to operate these Advices.

Trust is an important member of advice, and it is a very useful concept in the coordination in MAS, too ${ }^{5}$. Trust implies some form of risk, and that entering into a trusting relationship is choosing to take an uncertain path that can lead to either benefit or cost depending on the behavior of others. 
The perceived risk of cooperating with a particular SA-Agent is determined by that SA-Agent's reliability, honesty, etc., embodied by the notion of trust. Thus a SA-Agent can use its trust in others as a means of assessing the risk involved in cooperating with them. An inverse relationship between trust, $T$, and risk, $R$, is as follows.

$$
T=\frac{1}{R}
$$

In assessing the reliability of a parameter sent by others, a SA-Agent must make a judgment about the risk attached to cooperation with the sender, by examining the trust value in the Advice sent.

Suppose that a SA-Agent knows of $n$ others, SA-Agent, , SA-Agent $2, \ldots$ SA-Agent $_{n}$, with the required parameter for performing a situation assessment, and ordered such that $T_{S A-\text { Agent }_{t-1}} \geq T_{S A-\text { Agen }_{x}}$, where $T_{S A-A_{\text {gent }}}$ denotes the trust in SA-Agent $t_{x}$. Then the risk $R$ to take these advices (use the parameter) is:

$$
R=\frac{1}{\sum_{1}^{n} \frac{T_{S A-\text { Ageut }_{t}}}{i}}
$$

For a Battlefield Initiative computation or a situation prediction making with $m$ parameters, $a_{1}, a_{2}, \ldots, a_{m}$, the total risk $C$ for this situation assessment is:

$$
C=\sum_{i=1}^{m} R_{a_{i}}
$$

Now a result of a situation assessment $\omega$ (which may be a Battlefield Initiative or a situation prediction) and the risk $C$ related are gotten, so the Assessment Quality $Q$ can be given as follow:

$$
Q=\omega \times C
$$

Finally, through the system $\mathrm{L} / \mathrm{O}$, all these results are sent to the information bus, which carries all these results and other $\mathrm{C} 2$ information to Battle Commander, and carries the environment information to SA-Agents or to other entities in distributed $\mathrm{C} 2$ system, as presented in Fig 1.

By now a process of battlefield situation assessment is accomplished. With the change of battlefield, the system I/O takes the task of interchange information between the situation assessment system and the environment again, and a new assessment process begins.

\section{CONCLUSION}

To get a good assessment result needs integration of much technology. The agent-based approach in this paper gives us an intelligent environment 
to achieve this goal. SA-Agents can use the parameters in Advice, which gives not only the true number but also the Trust of these parameters. With these parameters, lots of technology whether is qualitative or quantitative can be integrated in this framework. And the more important is that with the help of SA-Agents, Commanders can get a clearer, more credible situation assessment for a better operation plan.

\section{REFERENCES}

1. Yang Fan, Study on Battlefield Situation Assessment in Command Automation of Air Unit, master thesis, (Air Force Engineering University, Xi'an, China, 2002).

2. Clinton Heinze, Simon Goss, and Adrian Pearce. Plan Recognition in Military Simulation: Incorporating Machine Learning with Intelligent Agents. In: Proceedings of the Sixteenth International Joint Conference on Artificial Intelligence, Workshop on Team Behavior and Plan Recognition, (Stockholm, Sweden, 1999), pp.53-63.

3. Wynn Stirling, A Model for Multiple Agent Decision Making, In: Proceedings of the 1991 IEEE Conference on Systems, Man, and Cybernetics. (New York, 1991), pp.2073--2078.

4. Mazda Ahmadi, Mehran Motamed and Jafar Habibi, Arian: A General Architecture for Advisable Agents, In: International Conference of Machine Learning; Models, Technologies and Applications (MLMTA'03), (Las Vegas, Nevada, 2003), pp.17-23.

5. Nathan Griffiths and Michael Luck, Cooperative plan selection through trust. In: MultiAgent System Engineering: Proceedings of the Ninth European Workshop on Modelling Autonomous Agents in a Multi-Agent World, edited by F. J. Garijo and M. Boman, (Springer, 1999), pp.162-174. 